La Revue

des Droits

de l'Homme

\section{La Revue des droits de l'homme}

Revue du Centre de recherches et d'études sur les droits fondamentaux

15 | 2019

Revue des droits de l'homme - $\mathrm{N}^{\circ} 15$

\title{
La représentation politique des femmes et la fragmentation du droit de vote et d'éligibilité : le cas taïwanais
}

Ying - Hsueh Chen

\section{(2) OpenEdition}

Journals

Édition électronique

URL : http://journals.openedition.org/revdh/5290

DOI : $10.4000 /$ revdh.5290

ISSN : 2264-119X

Éditeur

Centre de recherches et d'études sur les droits fondamentaux

Référence électronique

Ying - Hsueh Chen,

La représentation politique des femmes et la fragmentation du droit de vote et d'éligibilité :le cas

taïwanais », La Revue des droits de l'homme [En ligne], 15 | 2019, mis en ligne le 11 décembre 2018,

consulté le 10 juillet 2020. URL : http://journals.openedition.org/revdh/5290 ; DOI : https://doi.org/

$10.4000 /$ revdh.5290

Ce document a été généré automatiquement le 10 juillet 2020

Tous droits réservés 


\title{
La représentation politique des femmes et la fragmentation du droit de vote et d'éligibilité : \\ le cas taïwanais
}

\author{
Ying - Hsueh Chen
}

1 Afin de promouvoirl'accèsdes femmesau pouvoir,une variété demesures ont été adoptéesde par le monde, par exemple,

le fait de réserver des places aux femmes ou l'exigence de parité sur les listes électorales lors des élections politiques .L'article 134 de la Constitution de Taïwan(République de Chine ${ }^{1}$

) dispose dès son entrée en vigueur en 1947 que « dans les diverses élections politiques, le nombre de femmes à élire doit être prescrit par la loi ».Cette catégorie de « femmes »inscrite dans la Constitution et les quotas en matière électoraleen faveur des femmes

ont entrâné une fragmentation du droit d'éligibilité en construisant des catégories à

l'intérieur du peuple

, ce qui semble être contraire aucaractère universel des droitsfondamentaux. Selonles militantes pour les droits des femmesde l'époque, cette fragmentatio

$n$ était nécessaire pour garantir une véritable égalité dessexes quand une simple égalité devant la loi n'atteignait pas forcément lesobjectifsrecherché $\mathrm{s}$, à savoir la lutte contre les discriminations à l'égard des femmes et l'égalité hommesfemmes dans le milieu politique 
En Chine prise entre les conflits internes et la Seconde Guerre mondiale, les féministes commencèrent à se mobiliser contre l'exclusion des femmes de la vie politique et publique. Pendant les années 1930 et 1940, les négociations politiques furent

la République de Chine née en $1912^{2}$.

En l'occurrence, les féministes-suffragistes ont exigé que les femmes soient constitutionnellement considérées comme un groupe parmi les citoyens pour qu'elles

puiss

ent éliredes représentants des femmes à l'Assemblée nationale ${ }^{3}$

, comme d'autres groupes sociaux et ethniques minoritaires dans certaines régions.

Elles ont également exigé que des quotas électoraux pour les femmes soient intégrés dans la future constitution. Cela s'est passé avant que

la démocratie ne soit mise en œuvre et que les femmes n'obtiennent officiellement le droit de vote 4

. Leurs revendications ont finalement été entendues par les constituants et inscrites dans la Constitution de 1946. Ces exigences constitutionnelles ont ensuite été mises en place à Taïwan, suite à la fin de la colonisation japonaise en 1945 et au repli du gouvernement nationaliste chinois sur l'île en 1949

. Elles ont également fait l'objet de plusieurs réformes constitutionnelles visant à adapter la Constitution aux réalités propres de Taïwan. En tout cas, 1 a Constitution

de 1946 accorde une attention particulière à la condition des femmes et de certains groupes ethniques; $\mathrm{d}$

e plus, les Articles additionnels àla Constitution ${ }^{6}$, adoptésà lasuitede la démocratisation de Taïwan, imposentlareprésentation politiquede $s$ femmes ainsi que celle du peuple autochtonetaïwanais, et concrétisent des quotas électoraux en leur faveur.

3 La Constitution taïwanaise se caractérise ainsi par la prise enconsidération des groupes sociaux, dont celui des « femmes »,dans le domaine électoral , et installe un système de quotas de sièges réservés en leur faveur. Alorsque , de son côté,la Constitution françaisedémontre une conception universaliste des citoyenséligibleset une réticence vis-à-vis des catégories sociales, jusqu'à ce que , d'une part,la loi constitutionnelledu 8 juillet 1999ne soit venue questionner le modèle de l'universalisme républicainetintroduire la catégorisation des citoyens comme « hommes » et « femmes » ; et d'autre part, que les lois sur la parité en politique soient mises en place depuis 2000

Le fait que les femmes sont réduites à une catégorie sociale minoritaire parmi d'autres dans la Constitution de Taïwan éloigne notamment les expériences taïwanaises de la parité à la française qui pose l'irréductibilité de la bipartition hommes-femmes à d'autres catégories sociales à représenter. En effet, l'universalisme français républicain illustré par la décision du Conseil constitutionnel de 1982 rejette en principe toute reconnaissance de " catégories » ou mise en œuvre de « quotas » 
, présumées entraîner le risque du communautarisme et des revendications

particularistes

8

. Ainsi, la revendication de parité née à la fin des années 1980 a finalement été présentée en France comme une conséquence logique de l'universalité qui est celle de la différence de sexe, et comme l'instrument d'une meilleure représentation démocratique qui inclut les femmes constituant la moitié du genre humain. En outre, les arguments centrés sur l'égalité entre les deux sexes ont été relativement marginalisés 9 .

4 L'objectif de cette étudeest d'analyser l'élaboration mais aussil'application des mesures qui visent à promouvoir l'accès des femmes au pouvoir politique dans la Constitution de Taïwande 1946 et ses articles additionnels, tout en créant une catégorie à l'intérieur des citoyens. Elle tend également mais secondairement à montrer les spécificités dece

$s$ mesures d'action positive taïwanaises en les mettant en perspective avec la politique de « parité » à la française.

D'abord, la Constitution taïwanaise se caractériseparunecatégorisationdespersonnes éligiblesetdes électeurs

, à savoir les femmes et d'autres catégories sociales, ainsi que par une fragmentation du droit

de vote et d'éligibilité $\left(1^{\circ}\right)$.Ensuite, au niveau desa mise enœuvre , la fragmentation consiste en modalités desmesures d'action positive en faveur de la représentation politique des femmesqui varient selon le type d'élection mais sont toutes conçues en termes de résultats, à savoir des quotas au niveau des élus

\section{$1 \%$ - Fragmentation du droit de vote et d'éligibilité}

L'article 7 de la Constitution taïwanaise assure l'

égalité devant la loi de tous les citoyens sans distinction de sexe , de religion, de race, de classe ou d'affiliation politique. Pourtant, l'article 134 decette même Constitution, qui sera évoqué de manière plus précise ci-dessus,exige quel es quotasde sièges réservés auxfemmes soient prévuspour les élections politiques. En outre, selon l'article 26 de la Constitution concernant l'élection de l'Assemblée nationale, les organisations de sfemmes figurent parmi les groupes sociaux qui élisent leurs propres représentants dans cette institution.Il apparaît ainsidans la Constitutionde Taïwan une prise en compte des femmes en tant que catégoried'éligibles et d'électeurs dès le départ ; cela consacre un statut spécial aux femmes en matière électorale.

Il importe de rechercher pour quelles raisons et par quel processus furent-elles intégrées dans la Constitution ces mesures en faveur des femmes,malgré l'impératif du principe fondamental d'égalité

? La Constitution chinoise de 1946 contient deux approches différentes visant à garantir, et non seulement à promouvoir, l'accès des femmes aux postes électoraux. Celles-ci créent, d'une part, une fragmentation du droit de vote par les catégories des électeurs (A) 
et d'autrepart,

une fragmentation du droit de la représentation politique par les catégories des

éligibles (B).

\section{A/ - Fragmentation du droit de vote par les catégories des électeurs}

L'Assemblée nationale, organe souverain représentant le peuple, fut composée à la fois par les représentants des municipalités et ceux issus des " groupes » couvrant les forces révolutionnaires témoignant l'unité du pays, y compris les Mongoles et les Tibétans (alors indépendants), les minorités ethniques " en régions frontalières ", les Chinois d'outre-mer dits « expatriés », les groupes professionnels et ceux des femmes. Les femmes avaient le choix d'élire soit un(e) délégué(e) de leur municipalité en qualité de résidentes, soit une déléguée du groupe des femmes national ou provincial, cette fois-ci en qualité d'affiliées du groupe. La question vient notamment de la place des femmes dans cette catégorisation : comment les femmes furent-elles considérées comme « une des forces » à absolument présenter à l'Assemblée nationale ?

La revendication de l'égalité hommes-femmes est apparue en Chine continentale à la

siècle. Cette revendication et celle de l'émancipation des femmes faisaient partie de la propagande pendant la révol utionrépublicaine

visant à mettre fin à la dernière dynastie de la Chine impériale et à établir une

république

${ }^{10} . \mathrm{L}$

es mouvements pour les droits civiques des femmes se sont alors intensifiés lors de la proclamation de la République de Chine en 1911. Ils se sont poursuivis pendant

l'élaboration des Constitutions provinciales entre 1921 et 1923 et celle de la

Constitution provisoire en 1931

. Pendant cette période-là, les militantes, en insistant sur le fait que les femmes, en tant qu'être humain, sont identiques aux hommes, adoptèrent

des argumentaires tels que« les femmes sont également humaines

» ou « les droits de l'homme sont communs à tous, sansdistinction

entre les hommes et les femmes"

et revendiquèrent des droits égaux à ceux des hommes, y compris des droits civiques et le suffrage des femmes

${ }^{11}$.

8 Cependant,depuis le milieu des années 1930,

les revendications des campagnes pour les droits politiques des femmes ont changé : 
passant

de l'égalité hommes-femmes en droit à la reconnaissance des femmes comme groupe social ainsi qu'à l'établissement des quotas spéciaux en faveur des femmes dans les assemblées élues. Ce changement est accompagné d'un virage de stratégie rhétorique : l'activisme fondé sur l'identité hommes-femmes s'est transformé en un activisme fondé sur la différence entre les deux sexes. Néanmoins, la lutte pour la citoyenneté égale à celle des hommes n'est pas forcément identique à celle pour la représentation politique. La revendication d'un certain nombre de femmes représentantes siégeant aux assemblées élues ne se situe, de surcroît, pas forcément dans la continuité du combat pour le suffrage des femmes. La question qui se pose est donc de savoir d'où vient ce changement de revendications chez les militantes qui a finalement mené à l'intégration dans la Constitution des mesures en faveur de la représentation politique des femmes.

Le premier moment cruciala eu lieu en1924. À cette époque,la République de Chine, nouvellement proclamée, encore dépourvue d'

autorité centrale forte, fut dominée par les conflits entre les différentes factions de Seigneurs de la guerre.

Le chef du Parti nationaliste, Sun Yat-sen, en raison

de l'influence du socialisme depuis le mouvement du 4 mai(1919)

et de sa politique de coopération avec l'Union soviétiqueainsi qu'avec

le Parti communiste chinois

, en est venu dans les années 1920 à une conception différente de l'élection des représentants politiques.

En 1924, lorsqu'il proposa de convoquer une assemblée nationale, il suggéra que les délégués soient élus parmi différents groupes sociaux, par exemple, les partis politiques, les universités et les associations professionnelles, telles que celles des ouvriers et des agriculteurs

. Cette proposition créa un lien entre la représentation politique et l'appartenance aux groupes sociaux, etengendra chez les militantes des droits politiques des femmes la revendication de reconnaissance des femmes comme groupe social pouvant élire ses propres représentants à l'intérieur de cette future A ssemblée nationale. Ensuite,le projet de Constitution de 1936 prévitde constituer l'Assemblée nationale,chargée de l'électionduPrésidentde la Républiqueet $\mathrm{d}$ e la révision constitutionnelle, dereprésentants régionaux élus par les municipalités et communes,mais aussideconserver desquotas spéciaux pour la Mongolie, le Tibet,certains groupes ethniques etles " expatriés ", ressortissants chinoisà l'étranger.

En théorie, les femmes pouvaient également chercher à être élues comme représentantes

dans les municipalités ou communes mais aussiparmi ces groupessociaux. Néanmoins, celles-ci se préoccupaient dela quasi-absence des femmes dansces collectivités ou organisationscivilesainsi quedes inégalités entre hommes et femmes dans lesmilieux politiques et sociaux, notamment au niveau du pouvoir et des ressources. I l était peu probablepour les femmesd'entrer à l'assemblée. 
Afin d'assurer leur présence aux assemblées élues,exiger que les femmes soient également

considérées comme une catégorie qui élirait ses propres représentants parut être le seul moyen par lequel les femmes pouvaient avoir l'accès au pouvoir.Dans ces circonstances,

l'introduction d'une catégorie de « femmes » sur la liste de groupes qui élisent leurs propres représentants devint le nouvel objectif principal des militantes ${ }^{13}$.

D’après ces dernières, les femmes devaient être considérées comme un groupe aux besoins particuliers quand il s'agit d'élection, et devaient se voir accorder le même intérêtque lesgroupe s ethniques et les Chinois d'outre-mer lors de l'élection de l'Assemblée nationale ${ }^{14}$.

La participation au Conseil politique du peuple, Parlement provisoire en temps de guerre entre 1938 et 1948, donna aux militantes féministes la première occasion de l'action politique au niveau national .Elles étaienten effetnon seulement en mesure de faire du lobbying pour

les quotas spéciaux afin d'assurer la représentation politique des femmes, mais aussi d'influencer la révision du projet de Constitution de 1936. Le projet proposé par le

Conseil politique du peuple a finalement prévu qu'un quota spécial de femmes représentantes à l'Assemblée nationale soit établi pour les premières trenteannées après la promulgation de la Constitution, pour la raison qu'il serait difficile pour les femmes d'être élues sans ce soutien ${ }^{15}$.

Enfin, l'article 26dela Constitutionde Taïwande 194

6 prescrit que les membres de l'Assemblée nationalece sontd es représentants régionaux, ceux du Tibet et de la Mongolie,et ceux qui sont élus par d'autres groupes ethniques,les résidents à l'étranger, les organisations professionnelles et les organisations des femmes, dont le nombre reste à déterminer par la loi.Selon cette disposition, 1

es organisations des femmes figurent parmi d'autres groupes qui élisent leurs propres délégués de la même manière que les groupes ethniques et les organisations professionnelles. Par une catégorisation des électeurs et une fragmentation du droit de vote, cette disposition assurait d'une certaine manière la représentation de ces groupes.

\section{B/ - Fragmentation du droit de la représentation politique par les catégories des éligibles}

L'article 134 de la Constitution de Taïwan exige que l'élection d'un certain pourcentage de femmes soit garantie dans les diverses élections politiques, à l'exception de celle de l'Assemblée nationale, et serve de fondement de législation sur les quotas électoraux en faveur des femmes. Selon la Constitution et la loi électorale taïwanaises, les femmes sont ainsi considérées comme une catégorie d'éligibles. En construisant des catégories à l'intérieur du peuple, cette catégorie de «femmes » et les quotas en matière électorale entraînent une fragmentation du droit d'être élu.

La revendication des quotas en faveur des femmes, fondée principalement sur les
inégalités de fait et la différence entre hommes et femmes,


apparut parallèlement à celle de la reconnaissance des femmes comme groupe social qui élut ses représentants à l'Assemblée nationale

En effet, le principe d'égalité hommes-femmesa étéconfirmépar

la consécration du principe d'égalité dans le projet de Constitution du 5 mai 1936. De

plus,

les droitspolitiquesdes femmes ont également été reconnusselon ce document

. Autrement dit, le statut de citoyenneté des femmes a été officiellement assuréà ce moment.

15 À

partir de l'égalité hommes-femmes en droit nouvellement et officiellement acquise suite à

l'intégration du principe d'égalité dans le projet constitutionnel de 1936

, la réclamation d'une égalité réelle en termes de représentation politique par le biais des quotas électoraux en faveur des femmes est apparue. Le niveau d'éducation, la

position sociale

,les opportunités d'emploi des femmes, etc.étant beaucoup moins

avantageux que ceux des hommes, les femmes avaient des chances réduites de voir

leurs candidatures aux élections politiques présentées et soutenues

. Compte tenu des inégalités de fait entre les deux sexes dans tous les domaines de la société, les militantes féministes craignaient que les droits politiques des femmes etl'égalité hommes-femmesn'existent que

dans la lettre du texte. Pour elles, cette dernière

ne pourrait se réaliser qu'à la condition que les femmes, ayant plein droit de vote, puissent siéger au Parlement et aux assemblées élues. Les quotas en faveur des femmes leur

étaient donc nécessaires pour assurer la réalisation d'une véritable égalité hommes-

dans le domainepolitique ${ }^{16}$.

femmes

Suite à l'apparition deces

revendications, la différence entre les sexes fut adoptée comme stratégie rhétorique de

la campagne pour la représentation politique des femmes : ces dernières sont égales aux hommes, mais elles sont aussi différentes.

La guerre sino-japonaisequi éclataen 1937,

en pleine campagne pour les quotas électoraux en faveur des femmes, fournit aux militantes des opportunités différentes qu'elles n'auraient pas euesen temps de paix :

la guerre amplifia la notion traditionnelle de séparation des sphères et des rôles attribués aux hommes et aux femmes

,et la bipolarisation des

rôles attribués à chaque genre correspondait justement à l'argument employé par la campagne pour les quotas, à savoir la différence entre hommes et femmes 17.

Puis, lors de l'Assemblée constituante de 1946, les constituantes féministes continuèrent d'exiger que les quotas électoraux en faveur des femmes soient inclus dans la Constitution, pour que les femmes puissent participer à la prise de décisions politiques au même titre que les hommeset que 
l'égalité hommes-femmes en matière électoralesoit mieux réalisée. $\mathrm{L}$

es diverses propositions de modifications

concernant le chapitre de la Constitution sur les élections suggéraient : dans les

élections politiques, il fallait prévoir « au moins

trentepour cent » ou « au moinsvingtpour cent ${ }^{18}$

» de femmes élues, ou bien « le nombre de femmes élues est à prescrire par la loi » ${ }^{19}$

. En outre, concernantla compositionde l'Assemblée nationale,

les militantes insistèrent sur

l'ajout de représentants élus par les « organisations des femmes ».Dans toutes c

es propositions,les constituantes ontmontré

qu'il était peu probable pour les femmes de gagner lesélections, étant donné

les conditions socio-économiques désavantageuses dans lesquelles elles vivaient.

Par ailleurs, la première dame de la Républiquede Chine à l'époque,

Song Meiling, figurant parmi les constituantes qui soutenaient les quotas électoraux pour les femmes, a joué un rôle essentiel en exerçant une grande influence sur le vote des constituants du Parti nationaliste

${ }^{20}$.

Les efforts de cette époque se sont concrétisés avec,d'une part, l'inclusion des«

organisations des femmes »

dans la liste de groupes qui élisent leurs propres représentants à l'Assemblée nationale

(l'article 26 de la Constitution), et d'autre part, l'adoption générale des quotas

électoraux en faveur des femmes dans les élections des assemblées élues (l'article 134

de la Constitution). De plus, lors de l'élection parlementaire, le nombre des femmes

élues dans les différents scrutins devait également être

prescritpar la loi (l'article 64 de la Constitution).

La consécration des femmes comme une catégorie des électeursmais aussides éligibles

dans la Constitution de 1946reflète donc unefragmentation

du droit de vote et du droit de la représentation politique, quis'opposea priori

au caractère universel des droits

, mais visent en réalité à parachever l'universel des droits.

Contrairement àcette

conception quasi communautariste des électeurs et des éligibles de la Constitution de

Taïwan, ce qui a été retenu dans la Constitution française est un modèle du

citoyen abstraitet une conception universalistedes électeurs et des éligibles. ${ }^{21} \mathrm{C}$

e qui mène à la décision du 18 novembre 1982 du Conseil constitutionnel dans laquelle

ce dernier condamne l'introduction des « quotas par sexe »

dans les listes électorales et réaffirme

le principe d'indivisibilité des électeurs et des éligibles. Ainsi, 1

orsque les militantes chinoises exigent qu'un certain nombre de femmes siègent aux assemblées élues et que les quotas

de sièges réservéssoientinscrits dans la Constitution

pour que l'égalité hommes-femmes en matière électorale se réalise dans la pratique, le

droit

constitutionnelfrançaisprévoit

que le pouvoir doit être neutre du point de vue du genre : les gouvernants ne sont ni des hommes ni des femmes, mais des gouvernants

${ }^{22}$. Larevendication de la paritévisaitalorsà remédier àla 
situation de " tout masculin » sous l'universalisme,au nom duquel on avait refusé les droits de vote et d'éligibilité aux femmespar le passé.

Afin d'atteindre

un véritable universalisme non exclusif ainsi qu'une meilleure représentativité des gouvernants , la parité

hommes-femmes a été justifiée du point de vue d'une meilleure représentation politique, et présentée comme une conséquence de l'universalité vu que les femmes qui étaient exclues des lieux de pouvoir font la moitié du genre humain. Ce qui est tout le contraire de la stratégie de catégorisation chez les militantes chinoises. Néanmoins,

l'introduction de catégorie des femmes comme 1

a paritéhommes-femmes, elles sontun instrument parmi d'autres au service dela lutte contre ladiscrimination à l'égard des femmes dans l'accès à la vie politiqueet de l'égalité réelle entre hommes et femmes dans la sphère politique ${ }^{23}$.

Enfin, il fallait attendre la loi constitutionnelle du 8 juillet 1999 pour que le principe de « l'égal accès des femmes et des hommes aux mandats électoraux et fonctions électives » soit consacré dans la Constitution, que l'obstacle juridique établi par la décision du Conseil constitutionnel en 1982soit levé,et que les catégories des éligibles« hommes » et «femmes » puissent désormais être prises enconsidération par le législateur et les lois sur la parité mises en œuvre ${ }^{24}$.À travers ce mécanisme de parité, le constituant et le législateur français ontégalement accepté la distinction entre les candidats féminins et

masculins ainsi qu'une évolution de la définition traditionnelle de l'universalisme républicain.

${ }^{25}$ Les différentes conceptions constitutionnelles du citoyen et des catégories sociales impliquent des arguments et des revendications distincts chez les militantes chinoises et françaises des droits politiques des femmes avant l'élaboration ou la réforme de la

Constitution.

Lorsque les premières exigent que les femmes soient considérées comme un groupe qui possède des intérêts particuliers quant à la représentation politique

, les dernières insistent sur l'appartenance des femmes à

l'universel. Cependant, dans les deux cas, c'est face à l'exclusion durable des femmes de la sphère politique que les mesures de quotas ou de parité ont été revendiquées et mises en œuvre.

\section{$2^{\circ} \%$ - Fragmentation en modalités de mesures d'action positive en faveur des femmes en matière électorale variant selon le type d'élection}

Afin de promouvoir la représentation politique des femmes, d'une part, la Constitution de Taïwan décide, par ses propres dispositions, la composition de l'ancienne Assemblée

les « organisations des femmes » dans la listedes groupesqui

élisaient leurs propres représentants lors de l'élection de celle-ci (A). D'autre part, la

Constitution exige d'une manière générale que le législateur doive prévoir 1 es quotas en faveur des femmesdans les élections des assemblées élues 
. (B) Ces exigences ont fait l'objet de plusieurs réformes constitutionnelles mais aussi des révisions des lois électorales

depuis 1991

, l'année où a eu lieu la première élection de l'Assemblée nationale sur l'île de Taïwan, suite à celle de 1947 tenue en Chine.

\section{A/ - Organisations des femmes comme une catégorie d'él ecteurs lors de l'élection de l'Assemblée nationale}

Avant l'élaboration de la Constitution de 1946, les militantes des droits politiques des femmes ont exigé que ces dernières soient considérées comme un groupe social assimilable à d'autres groupes ethniques au moment des élections. Néanmoins, ce sont

« organisations des femmes

» qui ont finalement été choisies par les constituants comme catégorie des électeurs qui figuraient

dans la liste de groupes qui élisaient leurs propres représentants. Plus précisément, outre les

représentants régionauxélus par les municipalités et communes , ceux du Tibet et de la Mongolie,cette institution chargée à l'époque del'électiondu Présidentde la Républiqueet de la révision constitutionnelle étaitcomposée de représentantsélus par d'autres groupes ethniques, les résidents à l'étranger, les organisations professionnellesainsi que celles des femmes.

Lors de la première élection de l'Assemblée nationale en 1947, 168 représentants ont été élus par les organisations des femmes, dont 20 représentants élus par les organisations nationales, et 148 représentants élus par celles des provinces et des grandes métropoles

. Les candidats n'étaient pas limités aux membres des organisations des femmes. De plus, comme les citoyens disposent chacun d'une voix, si une personne faisait partie en même temps des organisations nationales et provinciales, elle ne pouvait participer qu'à une seule élection

27

. Par ailleurs, parmi les représentants régionaux et ceux des autres groupes, il y avait également des quotas pour les femmes prévus en raison notamment des exigences de l'article 134 de la Constitution sur les quotas électoraux en faveur des femmes 28.

Àla suite dela réforme constitutionnellede $1991^{29}$, une circonscription nationale au scrutin proportionnel a été créée pour l'élection de

l'Assemblée nationale. De plus, les organisations des femmes ainsi que les associations professionnelles ne constituent plus les groupes qui élisent leurs propres représentantsdans cette institution.Cependant,d es quotas pour les femmesétaientprévuspourles scrutins régionaux plurinominaux (des métropoles, communes et cantons) mais aussi pour le scrutin proportionnel au niveau national en suivantle principe d'un dixième : il fallait une femme élue pour tous les dix représentants élus. 
Plus précisément, lorsque le nombre de représentants à élire dans une commune ou le nombre d'élues d'un parti politique était entre 5 et 10, il fallait un siège réservé aux femmes ; lorsque le nombre dépassait 10, il fallait en ajouter un pour tous les 10 élus. Dans la circonscription nationale, les sièges sont attribués aux partis politiques selon le taux de voix remportées par chaque parti, compté à base des votes pour les candidats au scrutin plurinominaux. Le quotad'un dixièmea été élevé à un quart pour le scrutinproportionnel de la circonscription nationaleen $1997^{30}$.

Cela peut être considéré comme un résultat insatisfaisant suite aux revendications des militantes féministes d'augmenter à un quart les quotas électoraux en faveur des femmes à la veille de la révision constitutionnelle de 1997

${ }^{31}$. Enfin, c

ette assemblée s'est vue retirer le pouvoir de l'élection du président en 1992, année depuis

laquelle le président est élu directement par le peuple au scrutin uninominal majoritaire à un tour

. Elleaensuiteété abolie lors de la réforme constitutionnelle de 2005

, la révision constitutionnelle soumise désormais à la proposition du Parlement et au référendum

\section{B/ - Femmes comme une catégorie d'éligibles : des quotas électoraux en faveur des femmes}

L'article 134 de la Constitution de Taïwan

dispose que « dans les diverses élections politiques, le nombre de femmes à élire doit

être déterminé

par la loi

». Cette disposition sert de fondement aux législations électorales sur les quotas

de sièges réservés auxfemmes, et vise à garantir

la représentation politique des femmes au niveau des élus.

Il est généralement convenu que la présence de quotas électoraux en faveur des

femmes consacrés par la Constitution de 1946 est un traitement préférentiel spécial destiné à celles-ci en matière électorale.

Étant donné le statut social inférieur des femmes dans la société de la Chine ancienne

l'époque de l'élaboration de la Constitution,

depuis des siècles, à

l'égalité devant la loi n'assurait pas une compétition équitable entre les deux sexes. Dans ces conditions-là, l'objectif de la disposition mentionnée ci-dessus est de garantir qu'un certain nombre de femmes soient présentes et s'expriment dans les assemblées élues et que l'égalité effective entre hommes et femmes s'amplifie 32.

En ce qui concerne l'élection parlementaire, la composition duParlement

fut initialement prescrite à l'article 64 de la Constitution de 1946.

Selon cette disposition, c'est au législateur de prévoir les quotas en faveur des femmes dans les divers scrutins

del'élection parlementaire, y compris les scrutins régionaux

(de chaque province et grande métropole)et ceux 
organisant la représentativité du Tibet, de la Mongolie,desautres groupes ethniques, des résidents à l'étranger, etdes organisations professionnelles. Cet article a ensuite été remplacé par les a rticles additionnels à la Constitution

de 1991 suite à la première réforme constitutionnelle, depuis laquelle le parlement était composé de députés régionaux élus au scrutin plurinominal majoritaire , ceux élus dans la circonscription nationale au scrutin proportionnel, ainsi que ceux du peuple autochtone des plaines et des montagnes. Les citoyens ne disposaient de qu'une voix ; le vote unique au scrutin plurinominal pour les candidats servait également de celui pour les partis politiques au scrutin proportionnel. Les sièges étaient attribués aux partisenfonctiondu nombre de suffrages obtenus.Les a rticles additionnels à la Constitutionde 1991 ont préciséau niveau constitutionnel pour la première fois la modalité d'application des quotas pour les femmes enfixantle pourcentaged'un dixième pourles scrutins régionaux mais aussi celui de la circonscriptionnationale : il fallaitau moins une femme pour dix parlementaires élus ${ }^{33}$.

Lors de la réforme constitutionnelle de 2005, le nombre de parlementaires a été réduit de moitié, et l'article 4 des articles additionnels à la Constitution a instauré

un nouveau mode de scrutin pour le Parlement. Ce qui a mis en place un système mixte alliant scrutin uninominal majoritaire (73 députés à élire par 73 circonscriptions régionales, à savoir celles

des communes et des cantons

) et représentation proportionnelle (34 députés à élire au scrutin de liste dans la circonscription nationale

), avec 6 sièges réservés aux autochtones élus au scrutin unique non transférable. Chaque citoyen dispose désormais de deux voix : celle pour les candidats au scrutin uninominal et celle pour les partis politiques au scrutin proportionnel, respectivement. Cela dissocie la représentation régionale et nationale.

Pour la partie élue auscrutin uninominal, aucune mesure qui promeut l'accès des femmes en politique n'a étéprévue , ce qui peut être moins favorable aux femmes candidates. Au contraire, pour la partie élueau scrutinenreprésentation proportionnelle ${ }^{34}$ , le nombre de femmes ne peut être inférieur à la moitié sur chacune des listes des nomméssoumises par les partis politiques. Plus précisément, dans la circonscription nationale,des sièges seront attribués aux partis politiques qui présententdeslistes des candidats et qui obtiennent au moins 5 pour cent des voix,en proportion du nombre de suffrages remportés.Si le nombre de femmes nommées d'une liste est inférieur à ce qui est prescrit, les sièges seront attribués aux femmes sur les listes suivantes 35. 
Àla suitede

la première élection parlementaire en 1992 après la fin du régime autoritaire, les

femmes constituaient 10,56 \% des députés ; ce qui correspondait à l'exigence constitutionnelle alors en vigueur concernant le quota pour les femmes au Parlement, un dixième

${ }^{36}$. Ensuite, la proportion de femmes a augmenté au fur et à mesure ${ }^{37}$

. Les femmes représentaient30,09\% des députés après l'élection parlementaire de 2008 ${ }^{38}$,la première aprèsla réforme du mode de scrutin pour le Parlement en 2005 , contre $20,89 \%$ en $2004^{39}$.Elles représentaient $33,63 \%$ en $2012^{40}$.Après

l'élection de 2016, le pourcentage des députées atteint désormais 38,05 \% ${ }^{41}$.

Concernant l'électiondes organes législatifslocaux,

depuis 1950, les modalités d'élections locales étaient prescrites par les ordonnances administratives sur l'administration locale

42

. Cette situation persistait jusqu'en 1994, l'année où le Code sur l'administration locale de la Province et des communes ainsi que celui sur l'administration locale des métropoles ont enfin été élaborés. Selon ces ordonnances administratives, lors des élections de conseillers municipaux et cantonaux, il fallait au moins une femme élue pour 10 conseillers à élire. En outre, si le restant dépassait 5, il fallait également prévoir un siège réservé aux femmes.

Àla suite de la suppression de la« Province de Taïwan »

et d'autres réformes des collectivités territoriales en 1997, 1

e Code des collectivités localesa été mis en œuvre, etc'est lequotad'un quart quia été adoptét ${ }^{43}$.Ce Codeprescrit dès sa promulgationen 1999

que, dans les élections des conseillers municipaux et des représentants des cantons, « lorsqu'un total de 4 conseillers ou représentants est à élire dans une circonscription,

il doit y avoir une femme élue »

. Ensuite, 1

orsque le nombre total des conseillers ou des représentants à élire dépasse quatre, il doit y avoir une femme élue de plus pour tous les 4 élus supplémentaires . Le quota d'un quart adopté par le Codedes collectivités locales peut être considéré comme un résultat des revendications des militantes féministes qui exigent l'« augmentation des quotas électoraux », l'adoption du« quota d'un quart des sièges réservés pour les femmes »ou du« quota par sexe d'un tiers "

, depuis la veille des réformes constitutionnelles de 1997 et de 1999, lors desquelles ces quotas n'ont pas été intégrés dans les dispositions concernant les élections de

l'Assemblée nationale et du Parlement ${ }^{44}$.

31 Le quota de sièges réservés aux femmes et les modalités d'application des quotas électoraux varient en fonction dutype d'élections.

La fragmentation du droit de vote et d'éligibilité par la catégorie de femmes et le quota en faveur des femmes servent à mieux réaliser l'égalité dans la sphère politique. Il semble néanmoins que la fragmentation au niveau du choix de quotas manque de bienfondé. $\mathrm{L}$

esquotastelsqu'un dixième ou un quartontentraîné les doutes par rapport à sa légitimation etsa pertinence 
: Pourquoi ce taux-ci mais pas l'autre ? Ceux-ci devraient-ils correspondre à la proportion de femmes dans la population, de celles qui participent à la vie politique, ou de celles qui sont élues par le passé ? La question qui se pose est de savoir, dans un

fixé premier temps, si les pourcentages

s par le législateur aident à la réalisation de l'objectif cherché, à savoir l'égalité hommes-femmes en matière électorale ou même dans d'autres domaines ; et dans un second temps, si ceux-ci correspondent encore à la réalité. Néanmoins, 1

'adoption de tel ou tel quota n'était en général pas motivée ni justifiée dans les exposés des motifs des propositions lors de l'élaboration de la Constitution ou celles de révisions constitutionnelles.

En outre, en ce qui concerne la conformité aux réalités politiques des quotas de sièges réservés aux femmes, ces derniers ont également engendré le souci d'effet plafond pour les femmes.

Autrement dit, il se peut que les quotas électoraux en faveur des femmes deviennent une limite maximale du nombre de femmes élues. Cependant , selon les recherches empiriques, le nombre de femmes élues a dépassé le nombre prévu par la loi électorale depuis le début des années $1990^{45}$ . Les organisations ainsi que les militants pour les droits des femmes proposaient soit la suppression soit l'augmentation des quotas de sièges réservés aux femmes ${ }^{46} . \grave{A}$

la veille de la réforme constitutionnelle de 1997, ceux qui soutenaient l'augmentation de quotas électoraux ont insisté afin que, pour l'élection de l'Assemblée nationale mais aussi celle du Parlement, le quota en faveur des femmes soit augmenté à un quart

. Ensuite, avant la réforme de 1999, ils ont exigé que le quota par sexe d'un tiers soit adopté

48. De plus, depuis 2012, ils ont encore exigé que, pourl'électiondes organes législatifsloc aux, le quota de sièges réservés aux femmes d'un quart soit remplacé par le quota par sexe d'un tiers, et que l'objectif à moyen ou long terme d'un quota par sexe de 40 pour cent soit établi. Leurs revendications étaient fondées notamment sur la réalité électorale, à savoir le fait que les femmes ont de moins en moins été élues par le biais de sièges réservés, et que le nombre de femmes élues dans les diverses élections a dépassé les quotas prévus dans la Constitution et les lois électorales.

Pour fin de comparaison, lorsque la Constitution de Taïwan exige de prévoir qu'un certain nombre de femmes soit élu lors des élections politiques, la Constitution française vise, pour sa part, à favoriser l'égal accès des femmes et des hommes aux mandats électoraux.

De plus,les lois françaises sur la paritécherchent à assurer

une représentation équitable hommes-femmes au niveau des candidatures, la répartition équilibrée et sexuée des candidats.

De ce fait, afin de promouvoir la représentation politique des femmes, à Taïwan, les approches sont conçues en termes de résultats

, au niveau des élus, et l'objectif recherché consiste en une égalité en nombre qui n'était cependant qu'une garantie minimale pour les femmes ; a

lors qu'en 
France, les approches sont conçues en termes d'accès et l'objectif recherché consiste en la promotion d'une meilleure représentation politique, contrairement à celle qui était masculine à l'époque.

Plus précisément, les lois françaises

s'appliquent de manière contraignante pour les élections au scrutin de liste en imposant une obligation

d'alternance des candidats de chaque sexe sous peine d'invalidation des listes des candidats, et de manière incitative pour les élections législatives

au scrutin majoritaire uninominal en pénalisant financièrement les partis politiques qui ne respectent pas la parité des candidatures.

Comme la réforme

constitutionnelle de 1999 « favorise » mais ne garantit pas l'égal accès des femmes et des hommes aux mandats électoraux

le législateur peut être considéré comme n'étant tenu qu'à une obligation de moyens et non de résultats

49. Cela explique également

les limites de la loi du 6 juin 2000 qui incite mais ne contraint pas les acteurs politiques à respecter une égalité absolue dans la division du pouvoir entre les sexes 50 .

Lesdifférents contours de politiques d'action positive en faveur des femmes en matière électorale peuvent avoir pour origine le contexte historique dans lequel celles-ci ont été adoptées, la conception de l'égalité, celle de la représentation politique, le modèle de la citoyenneté, la position vis-à-vis de groupes sociaux et le mode de scrutin de chaque pays. Dans tous les cas, la présence plus nombreuse des femmes dans les lieux de pouvoir comble l'écart au niveau du pouvoir et des ressources mais secoue aussi la relation de pouvoir entre les sexes et déconstruit le stéréotype sexiste pour avancer vers l'égalité hommes-femmes.

$35 \grave{A}$

la veille de l'élaboration de la Constitution de 1946, les militantes féministes de Chine continentale exigèrent que les femmes soient considérées comme un groupe social qui élit ses propres représentants à l'Assemblée nationale, et que les quotas de sièges réservés aux femmes soient intégrés dans la future Constitution. Une telle revendication visait à assurer la présence des femmes dans les assemblées élues et la réalisation de l'égalité hommes-femmes en matière électorale garantie par le projet constitutionnel de 1936.

Suite aux revendicationset au lobbyingdes féministesau cours de cette période , les femmeschinoises ont officiellement gagné à la fois le droit de vote et d'éligibilité ainsi que la représentation politique dans une certaine mesure lors de l'inauguration de la Constitution de $1946^{51}$ : Les organisations des femmes 
figuraient parmi les groupes qui élisaient leurs représentants à l'Assemblée nationale ; en outre, le législateur devait prévoir les quotas électoraux en faveur des femmes pour toute élection politique. Autrement dit, 1

es approches adoptéespar les constituants

afin de remédier à l'exclusion des femmes de la sphère politique sont conçues au niveau des élus, à l'aide

de la catégorie de femmes mais aussides quotasspéciaux.

Cela paraît efficace et rassurant dans une période où la démocratie et l'égalité hommes-

femmes

étaienttout-justesmises en place.

Les mesures en cette matière ont ensuite été mises enœuvreà

Taïwan et les modalités de celles-civarient selon le type d'élection

. À la suite de cela, plusieurs réformes ont été mises en œuvre. Les femmes taïwanaises profitent de ce système de quotas en faveur des femmes intégré dans la Constitution lorsqu'il s'agit de la participation et de la représentation politiques. Le taux des femmes aux assemblées élues augmente alors au fur et à mesure. Néanmoins, celui-ci dépasse le quota prévu par le législateur depuis les années 1990 ; de plus, les femmes ont de moins en moins besoin de se faire élire par le biais de quotas spéciaux. Ainsi, la pertinence de pourcentage fixé par le législateur est mise en question et les propositions concernant

l'augmentation des quotas électoraux en faveur des femmes apparaissent à plusieurs reprises avant les révisions de la Constitution à la fin des années 1990. En outre, les

femmes issues de tout

milieu socio-économique

sont présentes aux assemblées élues, même si dans le cas de Taïwan c'est pratiquement

toujours les femmes de classe moyenne supérieure qui étaient engagées dans la

promotion de l'accès des femmes dans les lieux de pouvoir et celle de l'augmentation des quotas en leur faveur, avant l'élaboration mais aussi les réformes de la Constitution.

Le quota de sièges réservés aux femmes adopté par la Constitution de Taïwan améliore l'accès des femmes dans les lieux de pouvoir tout en évitant une fragmentation parmi le peuple. La catégorie de femmes et les quotas électoraux en leur faveur inscrits dans la Constitution, la fragmentation au niveau du droit d'éligibilité ainsi que la participation substantielle des femmes taïwanaises en politique n'ont pas mené à une fragmentation dans la société actuelle de Taïwan en créant une communauté de femmes ou bien une essentialisation de la catégorie de femmes à l'opposé de celle d'hommes. De plus, une telle communauté fondée sur le critère de sexe est d'autant moins réalisable qu'il existe déjà une grande hétérogénéité et diversité chez les femmes. Ce qui atténue les doutes vis-à-vis du quota par sexe et du quota en faveur des femmes en ce qui concerne les dangers du communautarisme.

Néanmoins, la question qui se pose alors est la suivante : puisque ne visant que les femmes, le système de quotas de sièges réservés, correspond-il encore à l'objectif constitutionnel de l'égalité réelle de genre ? E

n1992, les constituantstaïwanaisont consacré un paragraphe à l'article 10

des Articles additionnels à la Constitution

concernant les « politiques nationales fondamentales », qui dispose que «l'État

assure la protection de la dignité des femmes... et promeut l'égalité substantielle de genre ».

La question qui se pose est de savoirsi le système de quotasde sièges réservésne visant 
que les femmescorrespond encoreà l'objectif constitutionnel de l'égalitéréelle de genre . C'est le dilemme que rencontrent constamment la loi anti-discrimination et les discriminations positives : afin de remédier aux discriminations à l'égard des femmes et aux inégalités hommes-femmes, faudrait-il d'abord prendre en compte le sexe ? Recourrait-on aux mesures qui prennent en considération le sexe ( gender-conscious) ou aux politiques qui l'ignorent (gender-blind)?

En 1999, certains militants et organisations féministes ont commencé à suggérer de remplacer le système de sièges réservés aux femmes par les quotas par sexe (d'un tiers), c'est-à-dire les dispositions qui sont neutres du point de vue du genre 52.

En effet, les sièges réservés aux femmes visent à protéger les femmes et les considèrent comme défavorisées

, lorsque le quota par sexe cherche à réaliser la parité des genres ainsi que la justice de genre et celle au niveau de la distribution du pouvoir

54

. Du point de vue de ce dernier, les femmes sont défavorisées au niveau du pouvoir et des ressources, mais non au niveau de la capacité ; la position désavantageuse des femmes est une conséquence des discriminations sexuelles, mais non l'origine de la différence entre hommes et femmes. Si un système considère les femmes comme faibles, il perpétuera ou reproduira les discriminations envers les femmes dans la société. Par conséquent, le changement de position chez les militants pour les droits des femmes vis-à-vis des réformes constitutionnelles reflète leur effort de transformer l'image d $\mathrm{u}$ « besoin de protection » des femmes, mais aussi une autre conception de l'égalité de genre.

Lors des travaux préparatoires de la révision constitutionnelle de 2005, il y avait des propositions d'articles additionnels concernant les modalités d'élection parlementaire qui optaient pour le quota par sexe de 25 ou 30 pour cent et quiprescrivaient que«

la proportion d'élus de chaque sexe sur la liste de nommés des partis politiques au scrutin proportionnel ne peut être inférieur à un quart ou 30 pour cent $»^{55}$

. Néanmoins, ce qui a été intégré dans le projet de la révision constitutionnelle suite à la délibération parlementaire, et finalement été adopté par l'Assemblée nationale, c'est la disposition prescrivant que «

le nombre de femmes sur la liste de nommés des partis politiques ne peut être inférieur à la moitié

. Ainsi, en ce qui concerne les quotas qui favorisent la représentation politique du genre sous-représenté au Parlement, la dernière réforme constitutionnelle reste toujours dans la logique de mesures spéciales en faveur des femmes.

Enfin,il reste encore à savoir si ce genre d'avantage numérique accordé aux femmes en matière électorale, ou bien d'autres mesures neutres sur le plan du genre telles que les quotas par sexe et les lois sur la parité hommes-femmes, aident à améliorer l'égalité 
substantielle entre hommes et femmes, que cela soit dans la sphère publique ou professionnelle, ou dans la sphère privée.

\section{NOTES}

1. Le nom officiel de Taïwan reste la République de Chine, malgré son indépendance de facto ainsi que la «politique d'une seule Chine » voulue par la République populaire de Chine.

2. Il faut préciser que pendant cette période-là, l'île de Taïwan était encore sous l'occupation japonaise (1895-1945).

3. Sous la Constitution de 1946, celle-ci est l'équivalente du Collège électoral des États-Unis.

4. Dans certains soviets chinois, le système suffrage universel avec quotas du genre fut installé par des communistes. Qi WANG, «In tailwind, in headwind; Gender quotas in China », Indian Journal of Gender Studies, 22(3), p. 389.

5. À partir d'octobre 1949, la Constitution de la République de Chine ne s'applique que sur l'île de Taïwan et ses îles environnantes, même si au début celle-ci a été conçue pour appliquer à tout le territoire chinois. Entre le repli du gouvernement nationaliste à Taïwan en 1949 et la levée de l'état de siège sur l'île en 1991, la Constitution de 1946 fut partiellement «figée » et remplacée par les Dispositions temporaires applicables pendant la période de "rébellion communiste», sauf pour, inter alia, sa protection des candidates aux élections.

6. Ces « articles additionnels» révisent substantiellement la Constitution de 1946 en gardant le texte originel de celle-ci.

7. CC, Décision n 82-146 DC, 18 novembre 1982. Cette décision censure les dispositions d'une loi qui prévoyait de limiter à $75 \%$ le nombre de personnes du même sexe sur les listes des candidats aux élections municipales, en raison d'une violation du principe constitutionnel d'indivisibilité de la souveraineté.

8. Laure BERENI, Éléonore LÉPINARD, « Les femmes ne sont pas une catégorie » les stratégies de légitimation de la parité en France ", Revue française de science politique, 2004/1 (Vol. 54), p. 81.

9. Ibid., pp. 79-80.

10. Louise EDWARDS, «Women's suffrage in China: Challenging scholarly conventions ", Pacific Historical Review, 69(4), 2000, p. 620.

11. Louise EDWARDS, «From gender equality to gender difference: Feminist campaigns for quotas for women in politics, 1936-1947 », Twentieth Century China, 24(2), 1999, p. 74.

12. Hui-Ling KE, Gender and politics: Women in Modern Chinese Revolutionary Movement (1900s-1920s), 2004, pp. 197-199. Chang-Ling HUANG, «The formation of the politics of différence : Reserved seats for women in the 1946 Constitution ", Taiwanese Journal of Political Science, $\mathrm{n}^{\circ}$ 52, 2002, pp. 99-101.

13. Louise EDWARDS, « Realizing the power of différence: Quotas, war and elections ", in Gender, Politics, and Democracy: Women's Suffrage in China, Stanford University Press, 2008, p. 195-196.

14. EDWARDS Louise, «From gender equality to gender difference: Feminist campaigns for quotas for women in politics, 1936-1947 », op. cit., p. 88.

15. Hsiao-Ti LEE, «The constitutionality of reserved seats for women: From the perspective of the two principles of justice ", State, Constitution, Human Rights, Constitution Taiwan Foundation edited, Showwe Information, 2018, pp.271-272. Louise EDWARDS, «Realizing the power of différence : Quotas, war and elections », op. cit., pp. 216-217. 
16. EDWARDS Louise, «From gender equality to gender difference: Feminist campaigns for quotas for women in politics, 1936-1947 », op. cit., pp. 85-87.

17. Ibid., p. 81.

18. Le comité central du mouvement des femmes du Parti nationaliste adopta en février 1946 une résolution de promouvoir un quota électoral de $20 \%$ en faveur des femmes lors de l'Assemblée nationale consacrée à l'élaboration de la Constitution, étant donné que le taux des femmes qui entrèrent dans l'enseignement supérieur fut de $20 \%$, selon le ministère de l'Éducation. HsiuCheng HUANG, « Political participation of women in Taiwan after the World War II (1949-2004): The case of gender quota ", Asia Studies (52), 2006, p. 210.

19. Propositions $n^{\circ} 42, n^{\circ} 168, n^{\circ} 194, n^{\circ} 203, n^{\circ} 204, n^{\circ} 205, n^{\circ} 233, n^{\circ} 234, n^{\circ} 238, n^{\circ} 267$. Secretariat of National Assembly, Proceedings of the Constituent Assembly, 1946, pp. 809-810, 1031-1032, 1070-1074, 1077-1080, 1081-1082, 1082-1084, 1126-1127, 1127-1131, 1133-1135, 1164-1165.

20. Chen LEI, The making of the Constitution of the Republic of China: The constituent assembly, Daw Shiang Publishing, 2011, pp.156-160. Yen-wen PENG, 1999 Report on Taiwanese Women's rights: Political participation, Awakening Foundation, 1999, p. 82.

21. Olivia BUI-XUAN, Le droit public français entre universalisme et différencialisme, Economica, 2004, p. 16.

22. Isabelle BOUCOBZA \& Charlotte GIRARD, «La parité en politique: le genre, un outil de pouvoir », La loi et le genre : études critiques de droits français, CNRS éditions, 2014, pp. 509-510.

23. Ibid,, pp. 515-516.

24. Réjane SÉNAC-SLAWINSKI, La parité, PUF, 2008, pp. 73-74.

25. Dominiqie GILLOT, Vers la parité en politique, Paris, La Documentation française, 2001, p. 11.

26. Secretariat of Naitonal Assembly, Proceedings of the $1^{\text {st }}$ Naitonal Assembly, 1948, pp. 69-72.

27. Nan-Hai LEE, Studies on the election of the first Naitonal Assembly of 1947 after the implementation of the Constitution, Liberal Arts Press, 2013, pp. 62; pp. 242-245.

28. Secretariat of Naitonal Assembly, Proceedings of the $1^{\text {st }}$ Naitonal Assembly, op. cit., p. 42; pp. 45-68.

29. La révision constitutionnelle de 1991 est la première réforme après l'élaboration de la Constitution en 1946 et le repli du gouvernement nationaliste à Taïwan en 1949.

30. Nathan F. BATTO, Wen-Jong JUANG, Chiung-Chu LIN, "From one fourth to one third: The electoral effects of female reserved seats », Soochow Politics Journal, 32(1), 2014, pp. 102-103.

31. Chang-Ling HUANG, «From reserved seats for women to the gender quota system: Theory and practice of gender parity in politics », Issues \& Studies, $n^{\circ} 40(3), 2001$, p. 74.

32. Shuang-Lien LIANG, Yen-Ling KU, « Political participation of women in Taiwan: Observations within and beyond the regime", in The situation of women in Taiwan white paper, Taiwanese Feminist Scholars Association, China Times Publishing, 1995, pp. 97-98.

33. Lorsque le nombre de représentants à élire par chaque province et grande métropole ou le nombre d'élues d'un parti politique était entre 5 et 10 , il fallait un siège réservé aux femmes ; lorsque le nombre dépassait 10, il fallait en ajouter un pour tous les 10 élus.

34. En cela, le système électoral taïwanais se distingue du scrutin de liste de la France, puisque les citoyens votent pour les partis politiques qui présentent la liste des candidats et non une liste de candidats.

35. L'article 67 de la Réglementation sur l'élection parlementaire.

36. Wen-Chen CHANG \& Hsiao-Wei KUAN eds., Convention on the Elimination of All Forms of Discrimination against Women, Taiwan New Century Foundation, 2015, pp. 242-243.

37. Les femmes représentaient $14 \%$ des députés au Parlement en 1996, $19 \%$ en 1999 et $22 \%$ en 2002. 
38. En 2008, le pourcentage de femmes élues dans les circonscriptions régionales au scrutin uninominal majoritaire est de $28,07 \%$, et celui de femmes élues dans la circonscription nationale au scrutin proportionnel est de $50 \%$. Central Election Commission Database.

Election Results-Legislator Elections.

39. Chen-Yin JIANG, «The progress in women's political participation: From the point of view of female reserved seats ", Gender Equality Committee of the Executive Yuan, 2017.

40. En 2012, le pourcentage de femmes élues dans les circonscriptions régionales au scrutin uninominal majoritaire est de $25,32 \%$, et celui de femmes élues dans la circonscription nationale au scrutin proportionnel est de 52,94\%. Central Election Commission Database, Election ResultsLegislator Elections. «Women members of the 8th Legislative Yuan (Parliament of Taiwan, ROC) ", Gender Equality Committee of the Executive Yuan, 2012.

41. En 2016, le pourcentage de femmes élues dans les circonscriptions régionales au scrutin uninominal majoritaire est de 31,65\%, et celui de femmes élues dans la circonscription nationale au scrutin proportionnel est de 52,94 \%. Central Election Commission Database, Election ResultsLegislator Elections

"The gender composition of the 9th Legislative Yuan (Parliament of Taiwan, ROC) ", Legislative Yuan, 2018. "The gender composition of the 9th Legislative Yuan (Parliament of Taiwan, ROC)», Legislative Yuan, 2018

42. À cause de l'application des Dispositions temporaires, un amendement à la Constitution, pendant la période de rébellion communiste entre 1947 et 1991 ainsi que celle de la loi martiale entre 1949 et 1987, une variété de mesures ont été mises en place par l'ordonnance administrative sans fondement de la loi.

43. Paragraphe 5 de l'article 33 du Code des collectivités locales.

44. Joint declaration of feminist and social movement organizations, « Reserved seats for women of one quarter have fallen behind political reality; we demand that the gender quota of one third be integrated into the Constitution ", Awakening Magazine ${ }^{\circ}$ 203, Awakening Foundation, 1999. Hsin CHI, «The constitutional guarantee of one-quarter-reserved-seats-for-women is going to become an empty dream again?", Awakening Magazine $n^{\circ}$ 205, Awakening Foundation, 1999. Shih-chi HUNG, «One-quarter-reserved-seats for women with no ambition», Awakening Magazine $n^{\circ} 182$, Awakening Foundation, 1997. Chang-Ling HUANG, "From Reserved Seats for Women to the Gender Quota System : Theory and Practice of Gender Parity in Politics », op. cit., p. 74.

45. Par exemple, dans les scrutins régionaux de l'élection de l'Assemblée nationale de 1991, le quota en faveur des femmes était de 19 élues, mais il y avait 31 femmes représentantes élues. Chang-Ling HUANG, «From reserved Seats for Women to the Gender Quota System : Theory and Practice of Gender Parity in Politics », op. cit., p. 74. De plus, dans l'élection parlementaire de 1998, parmi 43 femmes élues, il n'y avait qu'une députée qui a été élue par le biais du système de quotas en faveur des femmes. Yen-wen PENG, 1999 Report on Taiwanese Women's rights: Political participation, op.cit., p. 82. Wen-Ting LU, «The Transformation on Political Participation of Women in Taiwan before the Abolition of Martial Law (1945-1987) », Taïwan Historica 62(1), 2011, pp. 273, 282. Nathan F. BATTO, Wen-Jong JUANG, Chiung-Chu LIN, «From one fourth to one third : The electoral effects of female reserved seats ", op. cit., pp. 102-103.

46. Hsiu-Cheng HUANG, «Political participation of women in Taiwan after the World War II (1949-2004): The case of gender quota », op. cit., 2006, p. 226.

47. Lors des réunions de l'Assemblée nationale, certaines Propositions des articles additionnels sur les quotas électoraux en faveur des femmes sont apparues. Par exemple, Proposition $n^{\circ} 57$ concernant la composition du Parlement exigeait qu'il fallait une femme élue pour tous les 5 élus lors de l'élection parlementaire. Proposition $n^{\circ} 77$ concernant l'article 134 de la Constitution sur les quotas électoraux en faveur des femmes exigeait que, dans toutes les élections des assemblées élues, le nombre de femmes élues ne pouvait être inférieur à un tiers. Proposition $n^{\circ} 114$ 
concernant également l'article 134 de la Constitution proposait le quota d'un quart. Proceedings of the $1^{\text {st }}$ meeting of the $3^{\text {rd }}$ National Assembly, Secretariat of Naitonal Assembly edited, 1997, pp. 346, 352,362 .

48. Joint declaration of feminist and social movement organizations, « Reserved seats for women of one quarter have fallen behind political reality; we demand that the gender quota of one third be integrated into the Constitution ", Awakening Magazine $n^{\circ} 203$, Awakening Foundation, 1999. Nathan F. BATTO, Wen-Jong JUANG, Chiung-Chu LIN, «From one fourth to one third: The electoral effects of female reserved seats », op. cit., p. 110.

49. Isabelle BOUCOBZA \& Charlotte GIRARD, «La parité en politique: le genre, un outil de pouvoir ", op. cit., p. 522.

50. Réjane SÉNAC-SLAWINSKI, La parité, op. cit., pp. 73-74.

51. Louise EDWARDS, "Women's suffrage in China: Challenging scholarly conventions », Pacific Historical Review, op. cit., pp. 617-619.

52. Chang-Ling HUANG, «From reserved seats for women to the gender quota system: Theory and practice of gender parity in politics », op. cit., pp. 77-80.

53. Il est indiqué dans l'exposé des motifs des Articles additionnels de 1991 que les quotas électoraux en faveur des femmes prévus pour les élections de l'Assemblée nationale et du Parlement étaient une " garantie minimum pour les femmes ». Secretariat of National Assembly, Proceedings of the first National Assembly, 1991, pp. 111-112.

54. Par exemple, dans l'exposé des motifs d'une Proposition des Articles additionnels pour la réforme constitutionnelle de 2005, il est indiqué que, pour que la répartition des sexes au Parlement soit conforme au principe de parité des genres, au scrutin proportionnel, le nombre de femmes sur la liste de nommés de chaque parti politique ne pouvait être inférieur à un tiers. Ce qui était nécessaire afin de promouvoir la participation politique de chaque sexe, et de réaliser l'égalité entre les sexes.

Documents of the $8^{\text {th }}$ assembly of the $5^{\text {th }}$ session of the $5^{\text {th }}$ Parlement, Proposition of additional articles of the Constitution $n^{\circ}$ 5468, 2004, pp. 137-138. Assembly Affairs Museum, Legislative Yuan.

55. Documents of the $6^{\text {th }}$ assembly of the $5^{\text {th }}$ session of the $5^{\text {th }}$ Parlement, Proposition of additional articles of the Constitution $n^{\circ} 5451,381-382$. Documents of the $8^{\text {th }}$ assembly of the $5^{\text {th }}$ session of the $5^{\text {th }}$ Parlement, Proposition of additional articles of the Constitution $n^{\circ} 5468,2004$, pp. 137-138. Parliament Report on the Proceedings and Results of Constitutional Amendment Propositions, 2005, pp. 1-6. Assembly Affairs Museum, Legislative Yuan.

\section{RÉSUMÉS}

Afin de promouvoir la représentation politique des femmes, des quotas électoraux en leur faveur ont été inclus dans la Constitution de Taïwan dès sa promulgation en 1947. Ainsi,les organisations de femmes figuraient parmi les groupes sociaux qui élisai ent leurs propres représentantsau sein de l'ancienne Assemblée nationale. Une telle prise en compte des femmescommecatégoriede personneséligibles et d'électeurs

dans la Constitution ainsi que les mesures qui favorisent l'accès des femmes dans les lieux de pouvoir

politiqueont entraîné une fragmentation du droitde vote etd'éligibilité en construisant des catégories à l'intérieur du peuple, malgré l'impératif du principe fondamental d'égalité. 
Par ailleurs, un phénomène de fragmentation est également perceptible dans les modalités de mise enœuvre de cesmesuresspéciales puisqu'ellesvarient selon le type d'élection

politique. Cependant, ces dernières sont conçues en termes de résultats, à savoir des quotas au niveau des élus, garantissant la présence d'un certain pourcentage de femmes dans les assemblées. Cette étude vise à

mettre au jour les raisons et le processus par lequel de telles mesures ont été intégrées dans la Constitution.

Elle tend également à montrer que celles-ci, encourageant la participation politique des femmes, buttent sur certaines difficultés telles que l'effet plafond pour le nombre d'élues, la mise en cause de la pertinence des quotas fixés par rapport à la réalité sociopolitique, et enfin, le doute qu'une politique publique ne favorisant que les femmes contribue à améliorer l'égalité substantielle de

genre.

In order to promote women's political representation, reserved seats for women are included in the Constitution of Taiwan since its promulgation.

Besides,women'

s organizations were one of the social groups that elect their own delegates in the former National Assembly.

The taking into consideration of women as a category of eligible candidates and electors, and the measures that further women

's participation in the political sphere both lead to

a fragmentation of the right to vote and eligibility,sincesuch considerations

establish categories inside the people, contrary to the imperative of the principle of equality.

In addition, the fragmentationcanalsobe found

in the modes of implementation of these special measures in favor of women'

s political representation which vary according to the type of political election. However, all of

them

are devised in terms of the results of election, namely at the level of elected representatives. The measures promoting women's political participation assure that a certain percentage of women be present in elected assemblies. This article aims to find out for what reasons and through what process they have been integrated in the Constitution, and tends to point out that this policy

leads to

such doubtsas whether itimposesalimitationon

the number of women representatives, whether the quotas fixed by the legislator correspond to political realities, and

whether a policy that

favors only women could really help to ameliorate substantial gender equality.

INDEX

Mots-clés : quotas, sièges réservés aux femmes, parité, action positive, discrimination positive, égalité hommes-femmes, représentation politique

Keywords : quotas, reserved seats for women, parity, affirmative action, gender equality, political participation 
AUTEUR

\section{YING - HSUEH CHEN}

Ying-Hsueh Chen est doctorant au Centre de théorie et d'analyse du droit, Université Paris Nanterre 